\title{
PATH FIELDS ON MANIFOLDS
}

BY

\author{
ROBERT F. BROWN(1)
}

Nash [11] defined a set $T_{0}$ consisting of paths $\alpha$ on a topological manifold $M$ such that for $0 \leqq t \leqq 1, \alpha(t)=\alpha(0)$ if and only if $t=0$. We add to this set the constant paths on $M$; call the union $T$ and give it the compact-open topology. The map $q: T \rightarrow M$ defined by $q(\alpha)=\alpha(0)$ is a fibre space in the sense of Hurewicz [10]. This fibre space extends the concept of the tangent bundle to topological manifolds in the sense that when $M$ is a differentiable manifold, $(T, q, M)$ is fibre homotopy equivalent to the tangent bundle of $M$ under a fibre homotopy equivalence which takes $T_{0}$ into the nonzero tangent vectors [6]. We recall that a vector field on a differentiable manifold may be defined as a cross-section in the tangent bundle. By analogy, we define a path field on a topological manifold to be a crosssection in $(T, q, M)$.

The principal result on the existence of nonzero vector fields on differentiable manifolds was discovered by Hopf [9]. The theorem states that a compact orientable differentiable manifold $M$ admits a vector field which is nowhere the zero vector if and only if the Euler characteristic of $M$ is zero. The purpose of this paper is to prove the corresponding theorem for path fields on topological manifolds: a necessary and sufficient condition for a compact orientable topological manifold to admit a path field which is nowhere the constant path is that the Euler characteristic of the manifold be zero.

A somewhat modified proof of the existence theorem, developed by Alexandroff and Hopf [1], is the model for the present proof. The main step in their development is the proof that every compact differentiable manifold admits a vector field which is the zero vector at one point at most. In other words, there is always a cross-section in the tangent bundle which intersects the standard cross-section of zero vectors at one point at most. This theorem, besides being of interest in its own right, reduces the problem from a global to a local one which is much easier

Presented to the Society, August 29, 1963; received by the editors September 9, 1963 and, in revised form, January 9, 1964.

(1) This paper is part of the author's doctoral dissertation written at the University of Wisconsin under the supervision of Professor Edward Fadell whose interest, patience and numerous helpful suggestions were of invaluable assistance in its preparation. The author also wishes to thank Professor Sufian Husseini for his encouragement and for many helpful discussions.

This research was supported by the National Science Foundation and by the Air Force Office of Scientific Research under grant AFOSR 90-63. 
to handle. We shall show in $\S 1$ that in a fairly large class of fibre spaces with standard cross-section over compact manifolds, a cross-section may be obtained which intersects the standard cross-section at one point at most. In particular, such a cross-section exists for the fibre space $(T, q, M)$ described above. In $\S 2$ we use this theorem together with the results of [4] to extend Hopf's existence theorem.

Hirsch [8] asked whether a topological manifold of Euler characteristic zero admits a fixed-point free map homotopic to the identity. The paper concludes with an affirmative answer to his question when the manifold is compact and orientable.

1. Cross-sections in generalized $n$-plane bundles. We shall use singular homology theory throughout this paper. We adopt the convention of omitting the coefficient group when it is the integers. For topological spaces $X$ and $Y$, we denote by $Y^{X}$ the space of maps from $X$ to $Y$ with the compact-open topology. Let $E^{n}$ be Euclidean $n$-dimensional space, let $S^{n-1}$ be the unit sphere in $E^{n}$, and denote by 0 the origin of $E^{n}$. Let $I$ stand for the unit interval $[0,1]$. If $X$ and $Y$ are topological spaces with $A \subset X, B \subset Y$, then $f:(X, A) \rightarrow(Y, B)$ is a map if $f \in Y^{X}$ and $f(A) \subset B$; we write that $f:(X, A) \rightarrow(Y, B)$ is a homeomorphism if $f$ is a homeomorphism of $X$ onto $Y$ and $f \mid A$ is a homeomorphism of $A$ onto $B$.

Let $(E, p, B)$ and $\left(E_{0}, p_{0}, B\right)$ be Hurewicz fibre spaces [10] over the same base space $B$. Fadell [6] defined $\left(E_{0}, p_{0}, B\right)$ to be a fibre subspace of $(E, p, B)$ provided $E_{0} \subset E, p_{0}=p \mid E_{0}$ and $(E, p, B)$ admits a lifting function $\lambda$ with the additional property that if $e_{0} \in E_{0}$ and $w \in B^{I}$ such that $p\left(e_{0}\right)=w(0)$, then $\lambda\left(e_{0}, w\right) \in\left(E_{0}\right)^{I}$. When $\left(E_{0}, p_{0}, B\right)$ is a fibre subspace of $(E, p, B)$, Fadell calls $\mathfrak{F}=\left(E, E_{0}, p, B\right)$ a fibred pair. When $\mathfrak{F}=\left(E, E_{0}, p, B\right)$ is a fibred pair, the fibre over $b \in B$ is the pair $\left(F, F_{0}\right)$ where $F=p^{-1}(b)$ and $F_{0}=p_{0}^{-1}(b)$. If $B$ is arcwise connected, the fibre is independent, up to homotopy type, of the choice of $b$.

Let $\mathfrak{F}=\left(E, E_{0}, p, B\right)$ and $\mathfrak{F}^{\prime}=\left(E^{\prime}, E_{0}^{\prime}, p^{\prime}, B\right)$ be fibred pairs over the same base space $B$. Fadell calls $\widetilde{F}$ and $\mathscr{F}^{\prime}$ fibre homotopy equivalent if there exist

(1) maps $\phi$ and $\psi$ such that the diagram

commutes,

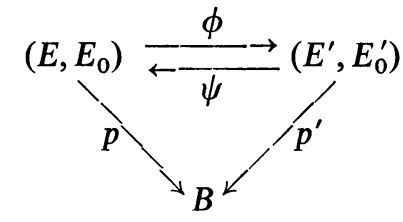

(2) a map $H:\left(E \times I, E_{0} \times I\right) \rightarrow\left(E, E_{0}\right)$ such that for all $e \in E, H(e, 0)=e$, $H(e, 1)=\psi \phi(e)$ and $H$ is fibre-preserving, i.e., $p H(e, t)=p(e)$ for all $t \in I$,

(3) a map $H^{\prime}:\left(E^{\prime} \times I, E_{0}^{\prime} \times I\right) \rightarrow\left(E^{\prime}, E_{0}^{\prime}\right)$ such that for all $e^{\prime} \in E^{\prime}, H^{\prime}\left(e^{\prime}, 0\right)=e^{\prime}$, $H^{\prime}\left(e^{\prime}, 1\right)=\phi \psi\left(e^{\prime}\right)$ and $H^{\prime}$ is fibre-preserving.

A cross-section of a map $p: E \rightarrow B$ is a map $\sigma: B \rightarrow E$ such that $p \sigma$ is the identity map on $B$. 
A generalized plane bundle (gpb) is a map $p: E \rightarrow B$ together with a crosssection $s: B \rightarrow E$ such that if $E_{0}=E-s(B)$ then

(1) $\left(E, E_{0}, p, B\right)$ is a fibred pair with fibre $\left(F, F_{0}\right)$,

(2) there is a homotopy $H: F \times I \rightarrow F$ such that $H\left(F_{0} \times[0,1)\right) \subset F_{0}$ and $H(F \times 1)=F \cap s(B)$.

A gpb is a generalized $n$-plane bundle ( $n$-gpb), $n \geqq 2$, if

(3) $F_{0}$ is arcwise connected and when $n \geqq 3, \pi_{1}\left(F_{0}\right)=0$, and

(4) $H_{*}\left(F, F_{0}\right) \cong H_{*}\left(E^{n}, E^{n}-0\right)$.

From property (2) we have that the fibre $F$ of a gpb is contractable, so for $k \geqq 1$, $H_{k}\left(F, F_{0}\right) \cong H_{k-1}\left(F_{0}\right)$. If $\mathfrak{F}=\left(E, E_{0}, p, B\right)$ is an $n$-gpb, we apply the Hurewicz Isomorphism Theorem to conclude that $F_{0}$ is $(n-2)$-connected and $\pi_{n-1}\left(F_{0}\right) \cong Z$ (the integers). This definition of $n$-gpb is slightly more general than that used by Fadell [6].

LEMMA 1.1. If $\mathfrak{F}=\left(E, E_{0}, p, B\right)$ is a fibred pair, $X$ is a topological space, and $f: X \rightarrow B$ is a map, then the induced fibred pair $f^{*}(\mathfrak{F})=\left(f^{*}(E), f^{*}\left(E_{0}\right), p^{*}, X\right)$ defined by setting

$$
\begin{aligned}
& f^{*}(E)=\{(x, e) \in X \times E \mid f(x)=p(e)\} \\
& f^{*}\left(E_{0}\right)=\left\{(x, e) \in X \times E_{0} \mid f(x)=p_{0}(e)\right\} \\
& p^{*}(x, e)=x
\end{aligned}
$$

is a fibred pair.

Proof. The fibred pair $\mathfrak{F}$ has a lifting function $\lambda$ from which we obtain a lifting function $\lambda^{*}$ for $f^{*}(\mathfrak{I})$ as follows. For $\omega \in X^{I}$ and $(\omega(0), e) \in f^{*}(E)$, let

$$
\lambda^{*}[(\omega(0), e), \omega](t)=(\omega(t), \lambda[e, f \omega](t))
$$

for all $t \in I$.

It is clear from the definitions that if $\mathfrak{F}$ is a gpb, then $f^{*}(\mathfrak{F})$ is also. Since the fibre of $f^{*}(\mathfrak{F})$ is homeomorphic to that of $\mathfrak{F}$, if $\mathfrak{F}$ is an $n$-gpb, so is $f^{*}(\mathfrak{F})$.

A well-known property of fibre spaces is that a fibre space over a contractable base is fibre homotopy equivalent to a trivial fibre space. By an extension of the same argument, the following result may be obtained.

Lemma 1.2 [6]. Let $\mathfrak{F}=\left(E, E_{0}, p, B\right)$ be a fibred pair and let $U \subset B$ be a set contractable in $B$ to a point $b_{0} \in U$. Then $\mathfrak{F} \mid U=\left(p^{-1}(U), p_{0}^{-1}(U), p, U\right)$ is fibre homotopy equivalent to a trivial fibred pair $\left(U \times F, U \times F_{0}, \pi, U\right)$ where $F=p^{-1}\left(b_{0}\right), F_{0}=p_{0}^{-1}\left(b_{0}\right)$ and $\pi: U \times F \rightarrow U$ is projection.

LEMMA 1.3. If $\mathfrak{F}=\left(E, E_{0}, p, B\right)$ is a gpb over a metric space $B$ and $U \subset B$ is a contractable in $B$ to a point $b_{0} \in U$ such that $b_{0}$ stays fixed throughout the contraction then there exists a fibre-preserving deformation retraction $R: p^{-1}(U) \times I \rightarrow p^{-1}(U)$ taking $p^{-1}(U)$ to $s(B)$ such that $R\left(p_{0}^{-1}(U) \times[0,1)\right) \subset E_{0}$ and $s\left(b_{0}\right)$ stays fixed throughout the retraction. 
Proof. Let

$$
\left(p^{-1}(U), p_{0}^{-1}(U)\right) \underset{\psi}{\stackrel{\phi}{\rightleftarrows}}\left(U \times F, U \times F_{0}\right)
$$

be the equivalence of 1.2 induced by the contraction of $U$. There is a fibre-preserving homotopy $G: p^{-1}(U) \times I \rightarrow p^{-1}(U)$ such that $G(e, 0)=e, G(e, 1)=\psi \phi(e)$ and $G\left(p_{0}^{-1}(U) \times I\right) \subset p_{0}^{-1}(U)$. Since $\mathfrak{F}$ is a gpb, there is a homotopy $H: F \times I \rightarrow F$ such that $H(e, 0)=e, H(e, 1)=s\left(b_{0}\right)$, and $H\left(F_{0} \times[0,1)\right) \subset F_{0}$. Let $C: U \times I \rightarrow B$ be the contraction of $U$ to $b_{0}$ (keeping $b_{0}$ fixed). For $b \in U$, define $C_{b} \in B^{I}$ by $C_{b}(t)=C(b, 1-t)$, then $\psi\left(b, b_{0}\right)=\lambda\left(b_{0}, C_{b}\right)$ (1). Define $\bar{\lambda}: E \rightarrow E^{I}$ by $\lambda(\alpha)$ $=\lambda(\alpha(0), p \alpha)$, then by Proposition 1 of [5], there is a fibre-preserving homotopy $K: E^{I} \times I \rightarrow E^{I}$ such that $K(\alpha, 0)=\bar{\lambda}(\alpha), K(\alpha, 1)=\alpha$ and from the proof of the proposition it is clear that if $\alpha(r) \in E_{0}$ then $K(\alpha(r), t) \in E_{0}$ for $t \in[0,1)$. Now define the deformation retraction $R$ of $p^{-1}(U)$ to $s(B)$ by

$$
R(e, t)=\left\{\begin{array}{lr}
G(e, 3 t), & 0 \leqq t \leqq 1 / 3, \\
\psi\left[p(e), H\left(\pi^{\prime} \phi(e), 3 t-1\right)\right], & 1 / 3 \leqq t \leqq 2 / 3, \\
K\left(C_{p(e)}, 3 t-2\right)(1), & 2 / 3 \leqq t \leqq 1,
\end{array}\right.
$$

where $\pi^{\prime}: U \times F \rightarrow F$ is projection. Since the functions agree at $t=1 / 3$ and

$$
\psi\left(p(e), H\left(\pi^{\prime} \phi(e), 1\right)\right)=\psi\left(p(e), b_{0}\right)=\bar{\lambda}\left(C_{p(e)}\right)(1)=K\left(C_{p(e)}, 0\right)(1),
$$

$R$ is continuous. The point $s\left(b_{0}\right)$ stays fixed throughout the deformation retraction because $B$ is metric and we may therefore take $\lambda$ to be regular [10].

The Covering Homotopy Theorem takes the following form in the theory of fibred pairs.

LEMMA 1.4. Let $E, B$ and $Z$ be topological spaces and let $E_{0}$ be a subspace of $E$. Suppose the following diagram commutes

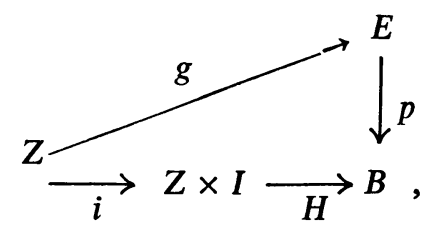

where $i(z)=(z, 0)$ and $g, p$ and $H$ are maps. Then $\left(E, E_{0}, p, B\right)$ is a fibred pair if and only if for each such diagram there is a map $G: Z \times I \rightarrow E$ such that $p G=H, G \mid Z \times 0=g$ and if $g(z) \in E_{0}$ then $G(z, t) \in E_{0}$ for all $t \in I$.

We will denote the boundary of a set $X$ (relative to a topological space containing $X)$ by $\partial X$.

Lemma 1.5. Let $\left(E, E_{0}, p, B\right)$ be $a$ gpb and let $c$ be a closed topological $n$-cell in $B$. We parametrize $c$ by a homeomorphism from the unit cell in $E$ onto 
so we can denote by 0 the point in the interior of corresponding to the origin of $E^{n}$. If there is a cross-section $\sigma^{\prime}: \partial c \rightarrow E_{0}$ then there is a cross-section $\sigma: c \rightarrow E$ such that $\sigma(c-0) \subset E_{0}$.

Proof. Consider the commutative diagram

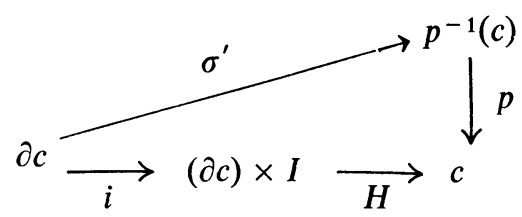

where $H(x, t)=(1-t) x$. Since $\left(p^{-1}(c), p_{0}^{-1}(c), p, c\right)$ is a fibred pair, there is a covering homotopy $\Sigma:(\partial c) \times I \rightarrow p^{-1}(c)$. Let $R$ denote the deformation retraction of 1.3 and define $f:(\partial c) \times I \rightarrow p^{-1}(c)$ by $f(x, t)=R(\Sigma(x, t), t)$. We observe that $p \Sigma(x, 1)=0$ so $f(x, 1)=0$ for all $x \in \partial c$. Therefore we may set $\sigma=f H^{-1}$.

Let $\mathfrak{F}=\left(E, E_{0}, p, B\right)$ be a gpb and let $\sigma: B \rightarrow E$ be a cross-section in $\mathfrak{F}$. If, for $b \in B, \sigma(b)=s(b)$, then $b$ is a singularity of the cross-section $\sigma$.

LEMMA 1.6. Let $\mathfrak{F}=\left(E, E_{0}, p, P ; F, F_{0}\right)$ be an $n$-gpb where $P$ is a finite connected n-dimensional polyhedron. Given $L, a$ subpolyhedron of $P$, and a crosssection $\sigma$ in $\left(p_{0}^{-1}(L), p_{0}, L\right)$, we may extend $\sigma$ to a cross-section in $\mathfrak{F}$ with a finite number of singularities.

Proof. Let $K=P-L$ and let $K^{m}$ be its $m$-skeleton. We may extend $\sigma$ to $\sigma: L \cup K^{0} \rightarrow E_{0}$ by sending a vertex $v$ into any point in $p_{0}^{-1}(v)$. Assume that $\sigma$ has been extended to $\sigma: L \cup K^{m} \rightarrow E_{0}, m<n-1$. Let $c$ be an $(m+1)$-simplex of $K$, then $\sigma$ is defined on $\partial c$ which is a topological $m$-sphere. Let

$$
p_{0}^{-1}(c) \underset{\psi}{\stackrel{\phi}{\rightleftarrows}} c \times F_{0}
$$

be a fibre homotopy equivalence and let $\pi: c \times F_{0} \rightarrow F_{0}$ be projection. Then $\pi \phi \sigma: \partial c \rightarrow F_{0}$ maps an $m$-sphere, $m<n-1$, into $F_{0}$ which is $(n-2)$-connected so $\pi \phi \sigma$ can be extended to a map $\Sigma^{\prime}: c \rightarrow F_{0}$. We define $\Sigma: c \rightarrow p_{0}^{-1}(c)$ by setting $\Sigma(x)=\psi\left(x, \Sigma^{\prime}(x)\right)$. We note that if $x \in \partial c$, then $\Sigma(x)=\psi \phi \sigma(x)$ so the fibrepreserving homotopy taking $\psi \phi$ to the identity induces a fibre-preserving homotopy $h^{\prime}:(\partial c) \times I \rightarrow p_{0}^{-1}(\partial c)$ such that $h^{\prime}(x, 0)=\psi \phi \sigma(x), \quad h^{\prime}(x, 1)=\sigma(x)$. Let $T=[((\partial c) \times I) \cup(c \times 0)] \subset c \times I$ and define a map $h: T \rightarrow p_{0}^{-1}(c)$ by setting $h$ equal to $h^{\prime}$ on $(\partial c) \times I$ and equal to $\Sigma$ on $c \times 0$. By the Fibre Homotopy Extension Theorem of [2], $h$ may be extended to a fibre-preserving homotopy $H: c \times l \rightarrow p_{0}^{-1}(c)$. Setting $\sigma(x)=H(x, 1)$ extends $\sigma$ to $c$. By repeating the same construction on each $(m+1)$-simplex of $K$, we can extend $\sigma$ to $L \cup K^{m+1}$. When $\sigma$ has been extended to $L \cup K^{n-1}$, we may apply 1.5 to each $n$-simplex of $K$ to obtain the required cross-section. 
The connectedness of $P$ in the last lemma was needed only to guarantee that the pre-image under $p_{0}$ of every point of $P$, which we indiscriminately called $F_{0}$, would be of the same homotopy type.

Let $X$ and $B$ be topological spaces, then $X$ dominates $B$ if there exist maps $f: X \rightarrow B$ and $g: B \rightarrow X$ such that $f g$ is homotopic to the identity map on $B$.

Employing the definition of covering dimension $n$, we may modify the proof of the Hanner Domination Theorem [7] to obtain:

Lemma 1.7. Let $B$ be an n-dimensional compact metric ANR, then $B$ is dominated by a finite $n$-dimensional polyhedron $X$ by means of maps $f: X \rightarrow B$ and $g: B \rightarrow X$ where $g$ is a barycentric mapping.

Lemma 1.8. Let $\mathfrak{F}=\left(E, E_{0}, p, B\right)$ be a fibred pair and let $X$ be a space which dominates $B$ by means of maps $f: X \rightarrow B$ and $g: B \rightarrow X$. The induced fibred pair $f^{*}(\mathfrak{F})=\left(f^{*}(E), f^{*}\left(E_{0}\right), p^{*}, X\right)$ admits a cross-section $\sigma^{*}$ if and only if $\mathfrak{F}$ admits a cross-section $\sigma$. If $\sigma^{*}$ is a cross-section in $f^{*}(\mathfrak{F})$ and $x \in X$ with $\sigma^{*}(x) \in f^{*}\left(E_{0}\right)$, then for a cross-section $\sigma$ in $\mathfrak{F}$ obtained from $\sigma^{*}$ we have that if $b \in B$ with $g(b)=x$, then $\sigma(b) \in E_{0}$.

Proof. The first part of the lemma is known [6]. We outline the proof here. If there is a cross-section $\sigma: B \rightarrow E$, define $\sigma^{*}: X \rightarrow f^{*}(E)$ by $\sigma^{*}(x)=(x, \sigma f(x))$. Given $\sigma^{*}$ we have

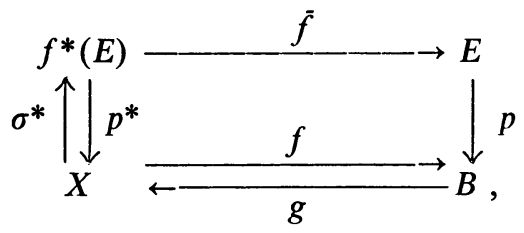

where $\bar{f}(x, e)=e$ and $p^{*} \sigma^{*}=$ id. Let $H: B \times I \rightarrow B$ be the homotopy connecting $f g$ and the identity. Define $\sigma^{\prime}: B \rightarrow E$ by $\sigma^{\prime}=\bar{f} \sigma^{*} g$, then $p \sigma^{\prime}=f g$ so $\sigma^{\prime}$ is a homotopy cross-section. By 1.4 there is a homotopy covering $H$ from which we may obtain a true cross-section for $\mathfrak{F}$. The second part of the lemma follows easily from 1.4 and the fact that $\bar{f}\left(f *\left(E_{0}\right)\right) \subset E_{0}$.

A connected separable metric space $B$ is an $n$-manifold if for each $b \in B$ there is an open subset $U$ of $B$ containing $b$ and a homeomorphism $h: E^{n} \rightarrow U$ (onto). The set $U$ is called a Euclidean neighborhood of $b$.

We shall assume throughout this paper that the dimension of all manifolds considered is at least two. The results are trivially true for lower dimensions, but separate arguments would be required.

LEMMA 1.9. Let $\mathfrak{F}=\left(E, E_{0}, p, B\right)$ be a gpb where $B$ is a manifold. If there exists a cross-section $\sigma^{\prime}$ of $\mathfrak{F}$ with a finite number of singularities, then there exists a cross-section $\sigma$ with at most one singularity. 
Proof. Let $x_{0}, \cdots, x_{q}$ be the singularities of $\sigma^{\prime}$. The proof of a theorem of $\mathrm{M}$. Brown and B. Casler [3] can be modified slightly to obtain the following result. Given an $n$-manifold $B$ and points $x_{0}, \cdots, x_{q}$ in $B$ there exists a map of the $n$-cell $C_{n}$ onto $B$ such that the restriction of the map to the interior of $C_{n}$ is a homeomorphism whose image contains $x_{0}, \cdots, x_{q}$. Thus the singularities of $\sigma^{\prime}$ are contained in a Euclidean neighborhood $V$ in $B$ and so there is a closed topological cell $C$ in $V$ containing $x_{0}, \cdots, x_{q}$ in its interior. By 1.5 there is a cross-section $\sigma$ over $C$ such that $\sigma\left|\partial C=\sigma^{\prime}\right| \partial C$ and $\sigma(z) \in E_{0}$ except at one point. Extend $\sigma$ to $B$ by setting it equal to $\sigma^{\prime}$ on $B-C$.

We are now in a position to prove the main result of this section.

THEOREM 1.10. If $\mathfrak{F}=\left(E, E_{0}, p, B ; F, F_{0}\right)$ is an $n$-gpb and $B$ is a compact $n$-manifold, then there exists a cross-section $\sigma$ of $\mathfrak{F}$ with at most one singularity.

Proof. Let $X$ be the dominating finite $n$-polyhedron of 1.7, then $f: X \rightarrow B$ induces an $n$-gpb

$$
f^{*}(\mathfrak{\Im})=\left(f^{*}(E), f^{*}\left(E_{0}\right), p, X ; F, F_{0}\right)
$$

Thus by 1.6 , there is a cross-section $\sigma^{*}$ in $f^{*}(\mathfrak{I})$ such that $\sigma^{*}(X)$ has a finite set of singularities $\left\{x_{i}\right\}$. Applying 1.8 we obtain a cross-section $\sigma^{\prime}$ for $\mathfrak{F}$ such that $\sigma^{\prime}(B) \cap s(B)=s\left(\bigcup g^{-1}\left(x_{i}\right)\right)$. The polyhedron $X$ is the nerve of an open cover of $B$, a manifold, so we may assume that the cover consists of Euclidean neighborhoods. From 1.5 we see that $x_{1}$ is in the interior of an $n$-simplex of $X$, so since $g$ is a barycentric mapping, $g^{-1}\left(x_{1}\right)$ is a compact subset of a Euclidean neighborhood and hence there is a closed topological cell $c$ in $B$ containing $g^{-1}\left(x_{1}\right)$ in its interior. Let $d^{\prime}$ denote the distance (in the metric of $B$ ) between $g^{-1}\left(x_{1}\right)$ and $\bigcup_{i>1} g^{-1}\left(x_{i}\right)$, let $d^{\prime \prime}$ be the distance from $g^{-1}\left(x_{1}\right)$ to $\partial c$ and set $d=\min \left(d^{\prime}, d^{\prime \prime}\right)$. Take a finite triangulation of $c$ of mesh less than $d / 3$, then no (closed) simplex of $c$ intersecting $g^{-1}\left(x_{1}\right)$ intersects a simplex which touches $\left(\bigcup_{i>1} g^{-1}\left(x_{i}\right)\right) \cup(\partial c)$. Let $P$ be the subpolyhedron of $c$ consisting of simplexes which do not intersect $\bigcup_{i>1} g^{-1}\left(x_{i}\right)$ and let $L$ be the subpolyhedron of $P$ consisting of simplexes which do not intersect $g^{-1}\left(x_{1}\right)$. We observe that $\sigma^{\prime} \mid L$ is a cross-section in $\left(p_{0}^{-1}(L), p_{0}, L\right)$. The polyhedron $P$ may not be connected, but $B$ is arcwise connected so $p_{0}^{-1}(b)$ is independent of $b \in B$ up to homotopy type. Thus by the remark following 1.6 , we may apply that lemma again to obtain a cross-section $\Sigma^{\prime}$ in $\left(p^{-1}(P), p, P\right)$ which is equal to $\sigma^{\prime}$ on $L$ and $\Sigma^{\prime}$ has only a finite number of singularities $\left\{b_{1, j}\right\}$. Extend $\Sigma^{\prime}$ to $B$ by setting it equal to $\sigma^{\prime}$ on $B-P$, then the singularities of $\Sigma^{\prime}$ are $\left\{b_{1, j}\right\} \cup\left(\bigcup_{i>1} g^{-1}\left(x_{i}\right)\right)$. If we repeat this construction for each $i>1$, we will obtain in a finite number of steps a cross-section $\Sigma$ of $\mathfrak{F}$ with only a finite number of singularities $\left\{b_{i, j}\right\}$. The application of 1.9 completes the proof.

Let $B$ be an $n$-manifold. We define (see [11])

$$
T_{0}=\left\{\alpha \in B^{I} \mid \alpha(t)=\alpha(0) \text { if and only if } t=0\right\} .
$$


Let $T \subset B^{I}$ be the union of $T_{0}$ and the constant paths on $B$ with the compactopen topology. Define $q: T \rightarrow B$ by $q(\alpha)=\alpha(0)$. A path field is a cross-section in $(T, q, B)$; a nonsingular path field on $B$ is a cross-section in $\left(T_{0}, q_{0}, B\right)$ where $q_{0}=q \mid T_{0}$.

THEOREM 1.11. Every compact n-manifold admits a path field with at most one singularity.

Proof. By 1.10 it is sufficient to prove that $\mathfrak{F}=\left(T, T_{0}, q, B ; F, F_{0}\right)$ is an $n$-gpb. The canonical cross-section consists of the constant paths on $B$. Fadell [6] showed that $\mathfrak{F}$ is a locally trivial fibred pair. The contraction $H: F \times I \rightarrow F$ is defined for $\alpha \in F, r, t \in I$ by $H(\alpha, t)(r)=\alpha((1-t) \cdot r)$. Fadell also proved that $\left(F, F_{0}\right)$ is the same homotopy type as $\left(E^{n}, E^{n}-0\right)$.

COROLlaRY 1.12. Let $B$ be a compact $n$-manifold, then there is a map $f: B \rightarrow B$ such that $f$ is homotopic to the identity map and $f$ has at most one fixed point.

Let $B$ be a differentiable $n$-manifold. A vector field is a cross-section in the tangent bundle $\left(E, p^{\prime}, B\right)$ of $B$. A nonsingular vector field is a cross-section in the bundle of nonzero tangent vectors $\left(E_{0}, p_{0}^{\prime}, B\right)$.

THEOREM 1.13 (Alexandroff and Hopf [1]). Every compact differentiable $n$-manifold admits a vector field with at most one singularity.

Proof. Fadell [6] has shown that when $B$ is a differentiable manifold, $\left(E, E_{0}, p^{\prime}, B\right)$ and $\left(T, T_{0}, q, B\right)$ are fibre homotopy equivalent, hence there is a fibre-preserving map $\phi:\left(T, T_{0}\right) \rightarrow\left(E, E_{0}\right)$. By 1.11 there is a path field $\sigma: B \rightarrow T$ with at most one singularity. If $\sigma(b) \in T_{0}$ then $\phi \sigma(b) \in E_{0}$ and $\phi \sigma: B \rightarrow E$ is the required vector field.

A generalized $n$-sphere bundle ( $n$-gsb), $n \geqq 1$, is a map $p: E \rightarrow B$ such that:

(1) $(E, p, B)$ is a Hurewicz fibre space with fibre $F$;

(2) $F$ is arcwise connected, and when $n \geqq 2, \pi_{1}(F)=0$;

(3) $H_{*}(F) \cong H_{*}\left(S^{n}\right)$.

If $\mathfrak{F}=\left(E, E_{0}, p, B\right)$ is an $n$-gpb, then $\left(E_{0}, p_{0}, B\right)$ is an $(n-1)$-gsb.

The proof of the following theorem follows step-by-step that of 1.10 and is therefore omitted. The only modification required is that if, for a cross-section $\sigma$ in the proof of $1.10, \sigma(B) \cap s(B)=s(Y)$, then, in the proof of 1.14 , that same $\sigma$ is defined only on $B-Y$.

TheOREM 1.14. Let $\mathfrak{F}=(E, p, B)$ be an $(n-1)$-gsb where $B$ is a compact $n$-manifold; then $\mathfrak{F}$ admits a cross-section except at one point at most.

2. Existence of nonsingular path fields. If $f, g \in Y^{X}$ with $f$ homotopic to $g$, then we write $f \sim g$. 
Let $M$ be an $n$-manifold and let $f: M \rightarrow M$ be a map. A point $x \in M$ is an isolated fixed point of $f$ if there is a Euclidean neighborhood $U$ of $x$ such that for $y \in U, f(y)=y$ if and only if $y=x$.

Consider the map $p: M \times M \rightarrow M$ given by $p\left(x_{1}, x_{2}\right)=x_{1}$ and let

$$
\Delta=\left\{\left(x_{1}, x_{2}\right) \in M \times M \mid x_{1}=x_{2}\right\} ;
$$

then Fadell proved [6] that $(M \times M, M \times M-\Delta, p, M)$ is locally trivial fibred pair, i.e., Fadell defined a homeomorphism $\zeta$ such that

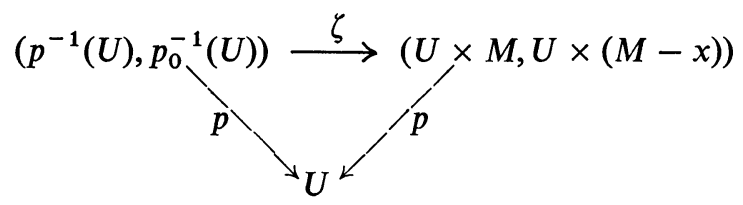

commutes. By excision, the inclusion $i:(U, U-x) \rightarrow(M, M-x)$ induces an isomorphism $i^{*}$. Let $\bar{\mu} \in H^{n}(M, M-x) \cong Z$ be a generator and let $i^{*}(\bar{\mu})=\bar{\mu}_{U}$. The map $f: M \rightarrow M$ induces

$$
(1 \times f):(U, U-x) \rightarrow\left(p^{-1}(U), p_{0}^{-1}(U)\right)
$$

by $(1 \times f)(y)=(y, f(y))$. Define $\pi: U \times M \rightarrow M$ by $\pi(u, y)=y$. Let $F^{*}$ be the homomorphism defined by the diagram

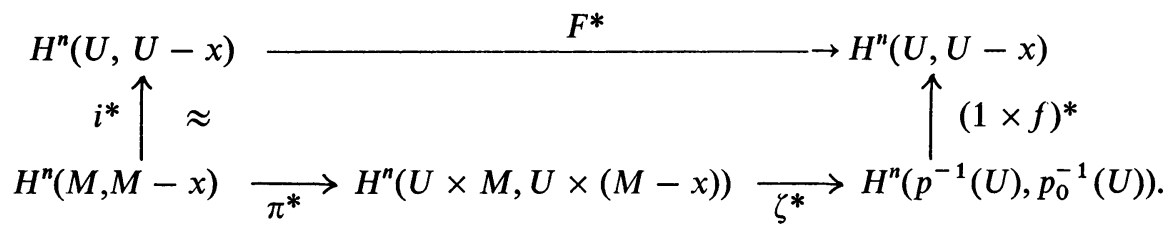

We define the index of the isolated fixed point $x$ of the map $f: M \rightarrow M, I_{f}(x) \in Z$, by $F^{*}\left(\bar{\mu}_{U}\right)=I_{f}(x) \cdot \bar{\mu}_{U}$. This definition of index is equivalent to the one given in [4] so it is well defined.

Define $f^{\prime}:(U, U-x) \rightarrow(M, M-x)$ by $f^{\prime}=\pi \zeta(1 \times f)$ and let $V$ be a Euclidean neighborhood of $x$ such that $V \cup f^{\prime}(V) \subset U$. There is a homeomorphism $h:\left(E^{n}, E^{n}-0\right) \rightarrow(V, V-x)$ and for $S=h\left(S^{n-1}\right) \subset V$ we know that setting $\bar{f}=f^{\prime} \mid S$ we have $\bar{f}(S) \subset U-x$.

LEMMA 2.1. Let $M$ be an n-manifold and let $f: M \rightarrow M$ be a map with an isolated fixed point $x$, then $I_{f}(x)=0$ if and only if $\dot{f}: S \rightarrow U-x$ is homotopic to a constant map.

Proof. Let $f^{\prime \prime}=f^{\prime} \mid V$. If $i:(U, U-x) \rightarrow(M, M-x)$ is inclusion, then by definition $F^{*} i^{*}=f^{\prime *}$ and for $j:(V, V-x) \rightarrow(U, U-x)$ the inclusion, $j^{*} F^{*} i^{*}=j^{*} f^{\prime *}=f^{\prime \prime} i^{*}$. Since $i^{*}$ is an isomorphism, $j^{*} F^{*}=f^{\prime *}$. Let $r: U-x \rightarrow S$ 
be a strong deformation retraction and let $k: S \rightarrow V-x$ be inclusion. From the exact cohomology sequence of $(U, U-x)$ we obtain the isomorphism

$$
\delta_{U}^{*}: H^{n-1}(U-x) \rightarrow H(U, U-x) .
$$

The preceding remarks show that diagram (1) commutes. If $\bar{f} \sim$ const : $S \rightarrow U-x$, then $\bar{f}^{*}$ is the zero homorphism so $F^{*}$ is also and $I_{f}(x)=0$. Conversely, if $I_{f}(x)=0$, then $F^{*}$ is the zero homomorphism and therefore so is $(r \bar{f})^{*}$. Thus the degree of $(r \bar{f}): S \rightarrow S$ is zero and by the Hopf Classification Theorem, $r \bar{f} \sim$ const. Let $k^{\prime}: S \rightarrow U-x$ be inclusion, then $k^{\prime} r f \sim$ const $: S \rightarrow U-x$. Since $k^{\prime} r \sim$ id: $U-x \rightarrow U-x$, we have $k^{\prime} r \bar{f} \sim \bar{f}$ and $\bar{f} \sim$ const.

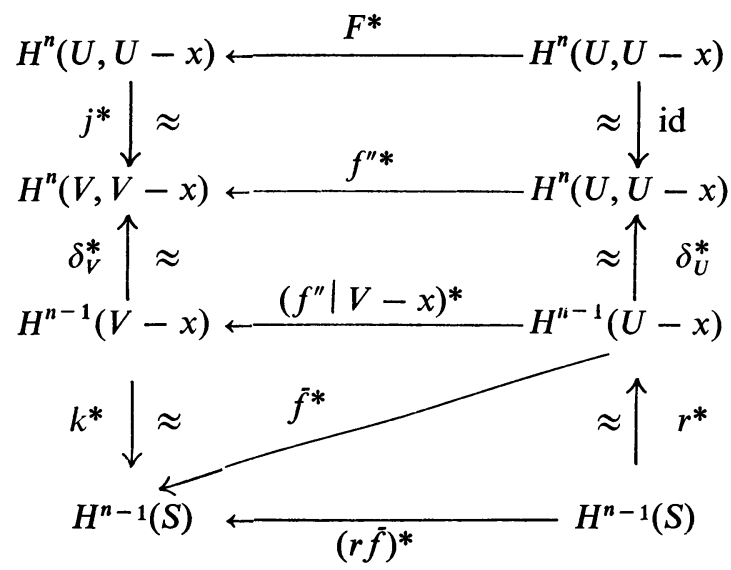

LEMMA 2.2. Let $M$ be an n-manifold and let $U$ be a Euclidean neighborhood of $x \in M$. Define

$$
F_{0}^{\prime}=\left\{\alpha \in U^{I} \mid \alpha(t)=x \text { if and only if } t=0\right\}
$$

and let $\psi^{\prime}: F_{0}^{\prime} \rightarrow U-x$ be given by $\psi^{\prime}(\alpha)=\alpha(1)$, then $\psi^{\prime}$ is a homotopy equivalence.

Proof. Let $h:\left(E^{n}, E^{n}-0\right) \rightarrow(U, U-x)$ be a homeomorphism and let

$$
E_{0}=\left\{\alpha \in\left(E^{n}\right)^{I} \mid \alpha(t)=0 \text { if and only if } t=0\right\} .
$$

Then $h$ induces a homeomorphism $h: E_{0} \rightarrow F_{0}^{\prime}$ given by $h(\alpha)(t)=h(\alpha(t))$. Define $\bar{\psi}: E_{0} \rightarrow E^{n}-0$ by $\bar{\psi}(\alpha)=\alpha(1)$ and since $\bar{\psi}=h^{-1} \psi^{\prime} h$, it is sufficient to show that $\bar{\psi}$ is a homotopy equivalence. Define $\bar{\eta}: E^{n}-0 \rightarrow E_{0}$ by $\bar{\eta}(y)(t)=t \cdot y$ then $\bar{\psi} \bar{\eta}=$ id. We observe that $\bar{\eta} \bar{\psi}(\alpha)(t)=t \cdot \alpha(1)$ and it is clear that if we define the homotopy $H: E_{0} \times I \rightarrow E_{0}$ by

then $\bar{\eta} \bar{\psi} \sim$ id.

$$
H(\alpha, r)(t)= \begin{cases}(t / r) \cdot \alpha(r), & 0 \leqq t \leqq r \leqq 1 \\ \alpha(t), & 0 \leqq r \leqq t \leqq 1\end{cases}
$$


THEOREM 2.3. A compact orientable n-manifold $M$ admits a nonsingular path field if and only if $\chi(M)=0$, where $\chi(M)$ denotes the Euler characteristic of $M$.

Proof. (Compare [6,3.8].) Let $\sigma: M \rightarrow T_{0}$ be a nonsingular path field. Define a map $f: M \rightarrow M$ by $f(y)=\sigma(y)(1)$. The map $f$ is fixed-point free so by the Lefschetz Fixed-Point Theorem, $\Lambda_{f}=0$, where $\Lambda_{f}$ is the Lefschetz number of $f$. Since $f$ is homotopic to the identity, $\Lambda_{f}=\chi(M)$ so $\chi(M)=0$ which proves that the condition is necessary. Conversely, if $\chi(M)=0$ we must construct a nonsingular path field on $M$. Consider the fibred pairs $\left(T, T_{0}, q, M\right),(M \times M, M \times M-\Delta, p, M)$ and define $\psi:\left(T, T_{0}\right) \rightarrow(M \times M, M \times M-\Delta)$ by $\psi(\alpha)=(\alpha(0), \alpha(1))$, then $\psi$ is fibre-preserving. By 1.11 there is a path field $\sigma^{\prime}: M \rightarrow T$ with one singularity; call it $x$. Let $U$ be a Euclidean neighborhood of $x$ and make the usual identifications

Let

$$
\left(p^{-1}(x), p_{0}^{-1}(x)\right)=(M, M-x) ;\left(q^{-1}(x), q_{0}^{-1}(x)\right)=\left(F, F_{0}\right) .
$$

$$
\zeta:\left(p^{-1}(U), p_{0}^{-1}(U)\right) \rightarrow(U \times M, U \times(M-x))
$$

be the homeomorphism defined by Fadell [6]. Define

$$
\zeta^{\prime}:\left(q^{-1}(U), q_{0}^{-1}(U)\right) \rightarrow\left(U \times F, U \times F_{0}\right)
$$

by $\zeta^{\prime}(\alpha)(t)=\zeta(\alpha(0), \alpha(t))$, then $\zeta^{\prime}$ is a homeomorphism such that

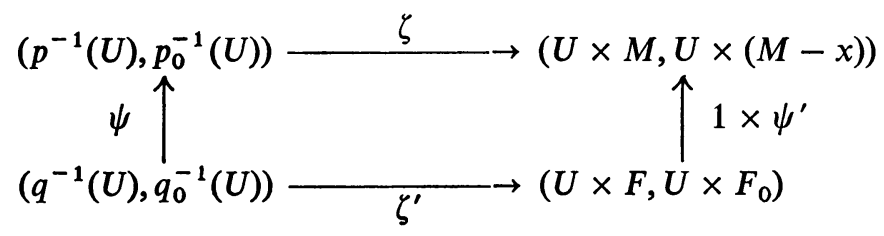

commutes, where $\left(1 \times \psi^{\prime}\right)(y, \alpha)=(y, \alpha(1))$ for $y \in U, \alpha \in F$. We define

$$
\Sigma=\pi \zeta^{\prime} \sigma^{\prime}:(U, U-x) \rightarrow\left(F, F_{0}\right),
$$

where $\pi:\left(U \times F, U \times F_{0}\right) \rightarrow\left(F, F_{0}\right)$ is projection. Since $\Sigma(x)$ is the constant path at $x$, there is a Euclidean neighborhood $V$ of $x$ contained in $U$ such that $\Sigma(y) \in U^{I}$ for all $y \in V$. Let $h^{\prime}:\left(E^{n}, E^{n}-0\right) \rightarrow(V, V-x)$ be a homeomorphism and set $S=h^{\prime}\left(S^{n-1}\right)$; then for $\bar{\sigma}=\Sigma \mid S$ we have $\bar{\sigma}(S) \subset F_{0}^{\prime}$. Let $f: M \rightarrow M$ be given by $f(y)=\sigma^{\prime}(y)(1)$ and define $(1 \times f): M \rightarrow M \times M$ by $(1 \times f)(y)=(y, f(y))$. Let $\bar{f}$ : $S \rightarrow M$ be defined by $\bar{f}=\pi \zeta(1 \times f) \mid S$, then

$$
\psi^{\prime} \bar{\sigma}=\psi^{\prime} \pi \zeta^{\prime} \sigma^{\prime}\left|S=\pi(1 \times \psi) \zeta^{\prime} \sigma^{\prime}\right| S=\pi \zeta \psi \sigma^{\prime}|S=\pi \zeta(1 \times f)| S=\bar{f}
$$

and since $\psi^{\prime}\left(F_{0}^{\prime}\right) \subset U-x$ we know that $\bar{f}(S) \subset U-x$ so the hypotheses of 2.1 are satisfied. By 2.2, $\bar{f}=\psi^{\prime} \bar{\sigma}$ implies that if $\bar{f} \sim$ const, so also is $\bar{\sigma}$. The map $f$ has $x$ as its only fixed point and $f \sim$ id. Since $M$ is compact and orientable (in the sense of [6]), by the results of [4], $I_{f}(x)=(-1)^{n} \Lambda_{f}=(-1)^{n} \chi(M)$. Therefore if $\chi(M)=0, \quad I_{f}(x)=0$ and by $2.1 f \tilde{f} \sim$ const: $S \rightarrow U-x$ which implies $\bar{\sigma} \sim$ const: $S \rightarrow F_{0}^{\prime}$. We recall the homeomorphism $h^{\prime}:\left(E^{n}, E^{n}-0\right) \rightarrow(V, V-x)$ 
and let $D^{n}$ be the unit cell in $E^{n}$ centered at the origin. If $C=h^{\prime}\left(D^{n}\right)$, then $S=\partial C$. Since $\bar{\sigma} \sim$ const : $S \rightarrow F_{0}^{\prime}$ we can extend $\bar{\sigma}$ to a map $\tau: C \rightarrow F_{0}^{\prime}$. Define the nonsingular path field $\sigma: M \rightarrow T_{0}$ by

$$
\sigma(y)= \begin{cases}\sigma^{\prime}(y), & y \in M-C, \\ \zeta^{\prime-1}(y, \tau(y)), & y \in C .\end{cases}
$$

COROLlaRY 2.4. Let $M$ be a compact orientable n-manifold, then there is a map $f: M \rightarrow M$ which is fixed-point free and homotopic to the identity if and only if $\chi(M)=0$.

Proof. If there is a map $f: M \rightarrow M$ which is fixed-point free and homotopic to the identity then $0=\Lambda_{f}=\chi(M)$. Conversely, if $\chi(M)=0$, then by $2.3, M$ admits a nonsingular path field $\sigma$ and we may define the map $f$ by $f(y)=\sigma(y)(1)$. Since $\sigma$ is nonsingular, $f$ is fixed-point free. The map $H: M \times I \rightarrow M$ given by $H(y, t)=\sigma(y)(t)$ is a homotopy connecting $f$ to the identity.

The preceding result answers the question of Hirsch [8] quoted in the introduction for this category of spaces.

Nash [11] proved that when $M$ is a differentiable manifold, $\left(T_{0}, q_{0}, M\right)$ is fibre homotopy equivalent to the bundle of nonzero tangent vectors on $M$, so the following result is a direct consequence of 2.3 (compare the proof of 1.13).

Corollary 2.5 (Hopf Existence Theorem [9]). A compact orientable differentiable manifold admits a nonsingular vector field if and only if $\chi(M)=0$.

\section{REFERENCES}

1. P. Alexandroff and H. Hopf, Topologie, Springer, Berlin, 1935.

2. G. Allaud and E. Fadell, A fibre homotopy extension theorem, Trans. Amer. Math. Soc. 104 (1962), 239-251.

3. M. Brown, A mapping theorem for untriangulated manifolds, The topology of 3-manifolds, Prentice-Hall, Englewood Cliffs, N. J., 1962.

4. R. Brown, On the Lefschetz fixed point formula, Amer. J. Math. (to appear).

5. E. Fadell, On fibre spaces, Trans. Amer. Math. Soc. 90 (1959), 1-14.

6. - Generalized normal bundles for locally-flat imbeddings, (to appear).

7. O. Hanner, Some theorems on absolute neighborhood retracts, Ark. Math. 1 (1951), 389-408.

8. M. Hirsch, Combinatorial and differentiable structures, Problem 18, Problems in differentiable and algebraic topology, American Mathematical Society, Summer Topology Institute, Seattle, 1963 (mimeographed).

9. H. Hopf, Vektorfelder in n-dimensionalen Mannigfaltigkeiten, Math. Ann. 96 (1927), 225-250.

10. W. Hurewicz, On the concept of a fibre space, Proc. Nat. Acad. Sci. U.S.A. 41 (1955), 956-961.

11. J. Nash, A path space and the Stiefel-Whitney classes, Proc. Nat. Acad. Sci. U.S.A. 41 (1955), 320-321.

UNIVERSITY OF CALIFORNIA,

Los Angeles, California 